GENETICS

\title{
Common co-deletion isn't a silent passenger
}

Methylthioadenosine phosphorylase (MTAP) is a ubiquitously expressed enzyme involved in the methionine salvage pathway. Homozygous deletion of MTAP is frequently seen in many human tumours, as the gene is located near to CDKN2A (which encodes cyclin-dependent kinase inhibitor 2A). Two studies in Science report that MTAP loss renders cells sensitive to inhibition of protein arginine methyltransferase 5 (PRMT5), a dependency that could be therapeutically targeted in MTAP-deleted cancers.

Kryukov et al. and Mavrakis et al. carried out large-scale short-hairpin RNA (shRNA)-mediated screens

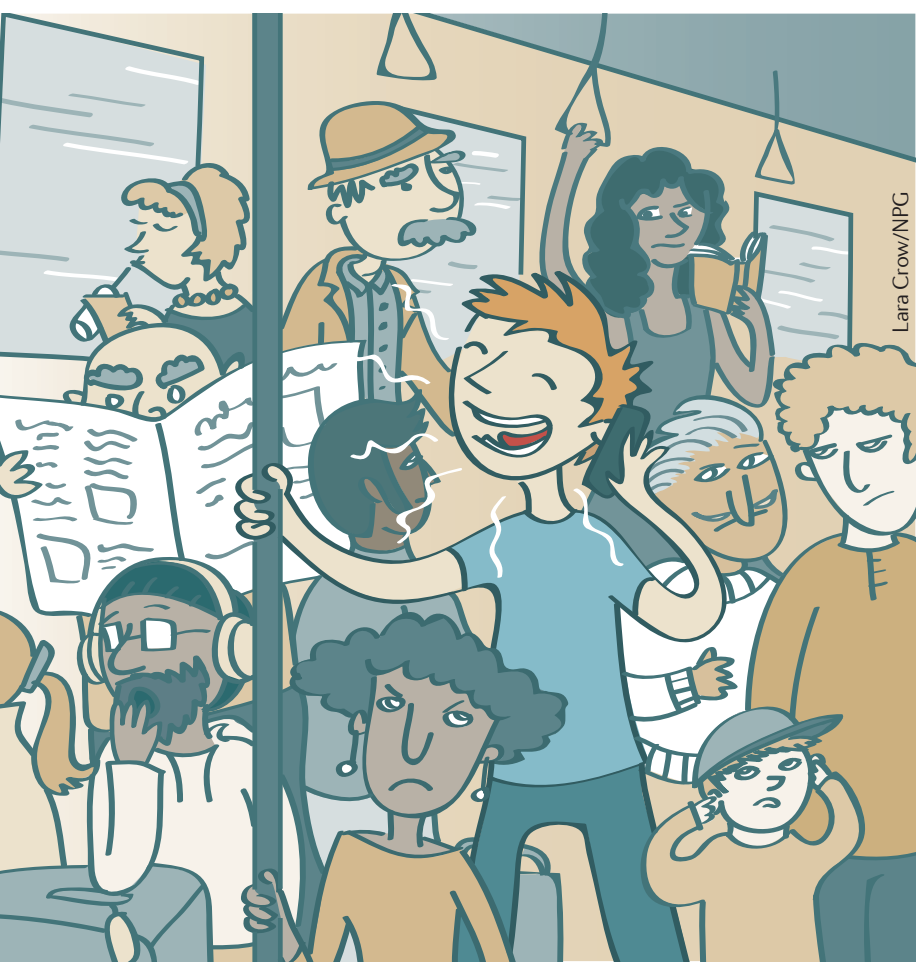

across cell lines from a range of cancer types to identify genes required for viability in $M T A P$-null but not $M T A P$ sufficient cells. For both groups, PRMT5 was the most significant hit.

Confirming that PRMT5 dependence is specifically induced by MTAP loss, cancer cell lines lacking $M T A P$ or both $C D K N 2 A$ and $M T A P$ were shown to be sensitive to PRMT5 knockdown, whereas those lacking only CDKN2A were not (Mavrakis et al.). Furthermore, sensitivity to PRMT5 knockdown was reduced by transducing four different $M T A P$-null cancer cell lines with MTAP (Kryukov et al.) and, conversely, was induced by CRISPR-mediated MTAP knockout in an MTAP-sufficient cancer cell line (Mavrakis et al.). PRMT5 activity was required for the effects of MTAP loss on cell viability, as the expression of catalytically inactive mutants of PRMT5 did not rescue the growth of MTAP-null cells in which PRMT5 was knocked down. In addition, using xenograft models of established disease induced by subcutaneous injection of mice with $M T A P$-null or MTAP-sufficient cancer cell lines engineered to inducibly express PRMT5-specific shRNAs, Mavrakis et al. demonstrated that in vivo PRMT5 knockdown markedly reduced tumour volume only in mice with MTAP-null tumours.

What mechanism links MTAP loss to PRMT5 dependence? Both groups investigated the role of methylthioadenosine (MTA), the substrate of MTAP, which had previously been suggested as a pan-PRMT inhibitor. Consistent with published data from studies analysing MTAP-deleted tumours, both groups found markedly increased levels of intracellular MTA in MTAP-null cell lines from various cancer types, a phenotype that was reversed by re-expression of MTAP. In addition, MTA levels were increased by CRISPR-mediated MTAP knockout in an MTAP-sufficient cancer cell line (Mavrakis et al.).

In line with the hypothesized role of MTA as a pan-PRMT inhibitor, increased intracellular MTA levels correlated with reduced catalytic activity of PRMT5 in MTAP-null cell lines (both groups). This correlation was not observed in isogenic MTAPreconstituted cell lines (both groups) but was observed in MTAP-sufficient cell lines treated with exogenous MTA (Kryukov et al.). Both groups used different assays to profile the ability of MTA to inhibit the catalytic activity of a panel of methyltransferases and showed that rather than being a pan-PRMT inhibitor, MTA is selective for PRMT5, and acts through a mechanism distinct from that of the PRMT5-selective inhibitor EPZ015666. Further characterizing the PRMT5 selectivity of MTA, crystal structure analyses revealed the key PRMT5 residues responsible for the conformational change induced by MTA binding (Mavrakis et al.).

These studies show that in the setting of MTAP loss, increased levels of MTA inhibit PRMT5 and render cancer cells sensitive to further PRMT5 inhibition. Thus, targeted therapies that exploit this vulnerability could be used to treat tumours in which MTAP is co-deleted with CDKN2A.

Lydia Shipman

ORIGINAL ARTICLES Kryukov, G. V. et al. MTAP deletion confers enhanced dependency on the PRMT5 arginine methyltransferase in cancer cells. Science http://dx.doi.org/10.1126/ science.aad5214 (2016) | Mavrakis, K. J. et al. Disordered methionine metabolism in MTAP/ CDKN2A deleted cancers leads to dependence on PRMT5. Science http://dx.doi.org/10.1126/ science.aad5944 (2016) 